\section{IDDF2019-ABS-0231 EFFECTS OF A MIXTURE OF THE SEED OF SYZYGIUM JAMBOLANA, FRUITS OF MOMORDICA CHARANTIA AND LEAVES OF AZADIRACHTA INDICA PASTE ON LOPERAMIDE-INDUCED CONSTIPATION IN RATS}

Nitin Kumar*, Rajender Khanna. Vivekananda Global University, India

10.1136/gutjnl-2019-IDDFabstracts.44

Background Constipation is one of the most common gastrointestinal complaints worldwide. Irritable bowel syndrome (IBS) is the most prevalent functional gastrointestinal disorders (FGIDs) that affects different aspects of life and patients experienced depression and anxiety more than others. The aim of this study is to evaluate the effects of a mixture of the seed of Syzygium jambolana, fruits of Momordica charantia and leaves of Azadirachta indica paste for the treatment of loperamide-induced constipation in a rat model.

Methods Animals were divided into one normal control group and three experimental groups $(10,20$ and $30 \mathrm{~g} / \mathrm{kg})$. Loperamide (2 $\mathrm{mg} / \mathrm{kg}$, twice per day) was injected intraperitoneally to induce constipation in the three experimental groups. Each group of rats was given orally a dose of granules containing (10, 20 and $30 \mathrm{~g} / \mathrm{kg}$ ) concentrated ethanolic extract of a combined mixture paste from all three folk plants. Mixture paste was administered for 30 days to assess its anti-constipation effects.

Results Fecal pellet number, weight, and water content were increased in the plant mixture paste-treated groups as compared to the control group. Reductions in body weight and increased intestinal transit length were observed in the plant mixture paste-treated groups. Fecal pellet number was reduced in the distal colons of the plant mixture paste-treated rats. Exercise and ileum tension increased in the experimental groups as compared to the control group. According to histological analyses, the thickness of the distal colon and areas of crypt epithelial cells that produce mucin were increased in the treatment groups in a dose-dependent manner.

Conclusions Constipation was decreased when combined plant mixture paste was fed to rats. Specifically, fecal number, weight, and water content, as well as histological parameters such as thickness and mucin areas in the distal colon were improved. A mixture of Syzygium jambolana, Momordica charantia and Azadirachta indica is effective in eliminating IBS symptoms, and it is a related useful therapeutic and preventive strategy for chronic constipation.

\section{IDDF2019-ABS-0235 METTL3 AS A POTENTIAL CANCER PROGNOSTIC MARKER PROMOTES THE PROLIFERATION AND METASTASIS OF GASTRIC CANCER CELLS}

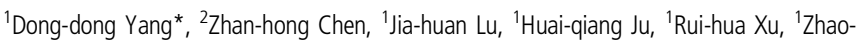
lei Zeng. 'Department of Medical Oncology of Sun Yat-sen University Cancer Center, State Key Laboratory of Oncology in South China, Collaborative Innovation Center for Cancer Medicine, China; '2Department of Medical Oncology and Guangdong Key Laboratory of Liver Disease, the Third Affiliated Hospital of Sun Yat-sen University, China

\subsection{6/gutjinl-2019-IDDFabstracts.45}

Background Gastric cancer serves as the fifth leading cause of malignancies, whose main cause of death is distant metastasis. Methyltransferase-like 3 (METTL3), a major component of N6methyladenosine (m6A) methyltransferase complex, has been suggested to function as an oncogene in several cancers. However, its clinical value and biological mechanism in gastric cancer remain unknown. Therefore, we attempted to investigate the expression profiles, prognostic value and possible downstream signal pathways of METTL3 in gastric cancer in this study.

Methods By analyzing data from the cancer genome atlas (TCGA), we depicted METTL3 expression profile and its possible downstream signal pathways in gastric cancer. Then, we further explored METTL3 expression and its prognostic values in 196 gastric cancers in our hospital. We established stable knockdown or overexpression of METTL3 gastric cancer SGC7901 cell lines to conduct in vitro and in vivo experiments.

Results METTL3 was significantly elevated in tumor tissues relative to normal gastric mucosa at both mRNA and protein levels. Moreover, the results from Kaplan-Meier survival curves analysis and multivariate Cox regression analysis demonstrated that METTL3 serves as an independent prognostic factor for gastric cancer patients. Furthermore, METTL3 can promote cell proliferation, colony formation, and cell migration and invasion. Additionally, the results of gene set enrichment analysis (GSEA) indicated that the potential downstream pathways of METTL3 were involved in cell cycle controlling. The top four pathways were as follows: the DNA repair pathway, the mitotic spindle pathway, the G2M checkpoint pathway and the E2F targets pathway.

Conclusions METTL3 was upregulated in gastric cancer and served as a promising prognostic biomarker for patients suffered this deadly disease. Moreover, METTL3 might play an oncogenic role in gastric cancer by the promotion of proliferation and invasion. The possible downstream pathways of METTL3 may be related to cell cycle controlling.

$\begin{array}{ll}\text { IDDF2019-ABS-0236 } & \text { PATTERNS OF USAGE OF SUGAR- } \\ & \text { SWEETENED BEVERAGES (SSBS) AND THE } \\ & \text { ASSOCIATED BEHAVIORS WITH } \\ & \text { EXPENDITURE INCURRED AMONG } \\ & \text { PERSONS VISITING THE GENERAL } \\ & \text { OUTPATIENT DEPARTMENT OF A TERTIARY } \\ & \text { CARE HOSPITAL }\end{array}$

${ }^{1}$ Shubhanshu Gupta*, ${ }^{2}$ Mani Goel. ${ }^{1}$ Department of Community Medicine, GMC Datia, India; ${ }^{2}$ Department of Pharmacology, MLBMC, Jhansi, India

10.1136/gutjnl-2019-IDDFabstracts.46

Background The burden of non-communicable diseases in India has already reached epidemic proportions. Sugar-sweetened beverages (SSBs) are implicated in causing obesity, diabetes and cardiovascular diseases. There is a lack of data from India regarding how frequently and how much sugary drinks are consumed, in what forms, what are the associated behaviors and expenditures incurred due to consumption of SSBs.

To document the prevalence and patterns of usage of SSBs, associated behaviors and expenditure incurred among persons visiting the general outpatient department of a tertiary care hospital. Methods This was a cross-sectional study conducted in a public tertiary care hospital located in Bhopal in central India, between May and September 2018. Patients and accompanying persons attending the general out-patient department and who were $\geq$ 15 years of age were included. Patients who are severely ill were excluded. Convenience sampling was used to select participants. Exit interviews using a semi-structured interview schedule were conducted with subjects giving informed consent and after patients had completed the physician consultation. 